\title{
Reduction of the Radiofrequency Heating of Metallic Devices Using a Dual-Drive Birdcage Coil
}

\author{
Yigitcan Eryaman, ${ }^{1}$ Esra Abaci Turk, ${ }^{1}$ Cagdas Oto, ${ }^{2}$ Oktay Algin, ${ }^{3}$ and Ergin Atalar ${ }^{1 *}$
}

In this work, it is demonstrated that a dual-drive birdcage coil can be used to reduce the radiofrequency heating of metallic devices during magnetic resonance imaging. By controlling the excitation currents of the two channels of a birdcage coil, the radiofrequency current that is induced near the lead tip could be set to zero. To monitor the current, the image artifacts near the lead tips were measured. The electric field distribution was controlled using a dual-drive birdcage coil. With this method, the lead currents and the lead tip temperatures were reduced substantially $\left[<0.3^{\circ} \mathrm{C}\right.$ for an applied $4.4 \mathrm{~W} / \mathrm{kg}$ SAR compared to $>4.9^{\circ} \mathrm{C}$ using quadrature excitation], as demonstrated by phantom and animal experiments. The homogeneity of the flip angle distribution was preserved, as shown by volunteer experiments. The normalized root-mean-square error of the flip angle distribution was less than $10 \%$ for all excitations. The average specific absorption rate increased as a trade-off for using different excitation patterns. Magn Reson Med 69:845-852, 2013. (c) 2012 Wiley Periodicals, Inc.

Key words: RF safety; transmit arrays; dual-drive birdcage coil; implant heating

\section{INTRODUCTION}

In general, for both diagnostic and interventional MRI procedures involving long, wire-shaped conductor devices, such as catheters, biopsy needles, and active implantable medical devices, amplification of the specific absorption rate (SAR) at the device tip poses a potential risk for patient safety. SAR amplification can cause tissue burns if it is not managed with appropriate methods $(1,2)$. Different methods have been reported for modifying the device geometry or adding lump circuit elements to the device to reduce the tip SAR amplification (3). However, such modifications of the device geometry or circuitry could reduce the mechanical robustness. In addition, for certain applications, such as biopsy procedures, it is not feasible to modify the metallic needles with the above

${ }^{1}$ Department of Electrical and Electronics Engineering, Bilkent University, Ankara, Turkey.

${ }^{2}$ Faculty of Veterinary Medicine, Department of Anatomy, Ankara University, Ankara, Turkey

${ }^{3}$ Department of Radiology, Ataturk Training and Research Hospital, Ankara, Turkey.

Grant sponsor: TUBITAK; Grant number: 107E108.

${ }^{*}$ Correspondence to: Ergin Atalar, Ph.D., National Research Center for Magnetic Resonance (UMRAM), Department of Electrical and Electronics Engineering, Bilkent University, 06800 Ankara, Turkey. E-mail: ergin@ee. bilkent.edu.tr

Received 4 October 2011; revised 26 February 2012; accepted 9 April 2012.

DOI 10.1002/mrm.24316

Published online 10 May 2012 in Wiley Online Library (wileyonlinelibrary. com).

(c) 2012 Wiley Periodicals, Inc. methods. Furthermore, for patients who carry MR-incompatible devices, replacing the unsafe devices with the safer ones may not always be practical. Finally, when the economic costs of these modifications are considered, determining a solution related to MRI aspect of the problem may be more reasonable.

In our previous study, we proposed a method based on the use of linearly polarized birdcage coils (4) to minimize radiofrequency ( $R F$ ) heating at the wire tips. Because the electric field of the linear birdcage coil is equal to zero on an entire plane, the location of the metallic device was coincided with this plane, and the RF heating was reduced. Using this approach, the homogenous transmit sensitivity characteristics were preserved. At 3.0 Tesla, this technique worked well in uniform phantoms. However, tissue inhomogeneities can alter the electric field distribution in the body. In this case, knowing the coil geometry and the conductor currents in the coil may not be sufficient to estimate the location of the zero-electric-field plane. The shape of the zeroelectric-field plane can be distorted, or its position can be shifted in a manner that is difficult to estimate. In general, when scanning patients with metallic devices using linear polarized excitation, monitoring the local electric field experienced by the metallic device is crucial. Therefore, the device can be coincided with the zero-electric-field zone of the linear coil, and the patient can be scanned safely. However, if a portion of the medical device lies outside of the zero electric field, a risk remains for RF current to be induced in the leads of the device.

In the literature, different methods based on measuring RF-induced currents directly by attaching optoelectronic circuits to the conductor leads are described (5). Although accurate measurements can be performed using these methods, when the modification of the metallic device is not possible, these approaches cannot be used noninvasively. There also exist other methods, in which the magnitudes of the RF currents on the metallic wires are calculated from their artifacts in MR images (6). These artifacts can also be used to monitor the level of RF-induced currents on the leads.

In another study (7), transmit arrays were used to control the induced currents on a guide-wire conductor; the relationship between the excitation voltages and the induced currents on the lead was modeled as a linear system, and the null space of the excitation matrix was computed. This approach enabled the induced current to be set to zero on the lead. However, in that study, the homogeneity of the flip angle distribution in the body due to transmit array excitation was not considered. The excitation that minimizes the induced current on a lead should also be convenient for performing imaging with a homogenous flip angle distribution. 
In this study, we used a dual-drive birdcage coil to determine a generic solution to reduce the induced current near the tip of a metallic implant lead. By changing the magnitude of the excitation currents on two separate channels of the coil, the electric field pattern was modified to determine a safe excitation pattern that minimizes RF current artifacts near the lead tip. To monitor the current, the image artifacts near the lead tips were measured. Phantom experiments were performed with copper wires and Deep Brain Stimulator (DBS) leads. To demonstrate the feasibility of the proposed method in the presence of tissue inhomogeneity, an animal experiment was performed. The device tip temperatures were reduced substantially with respect to standard quadrature excitation. The performance of the method was tested for different lead geometries. For some lead geometries, linear polarized excitation could be used to reduce the maximum increase in the tip temperature to less than $0.3^{\circ} \mathrm{C}$ for an applied $4.4 \mathrm{~W} / \mathrm{kg}$ SAR. With linear excitation, the homogeneity of the flip angle distribution was preserved compared with quadrature excitation. Finally, for several other lead geometries, elliptical polarization was required to reduce the temperature at the tip.

\section{THEORY}

The electric field distribution due to a linear and uniform excitation, in a cylindrical homogenous object that is infinitely long in the $z$ direction can be expressed as follows (4):

$$
E_{z 1}=-H_{f} j \omega \mu_{0} \rho \sin \phi, E_{\phi 1}=0, E_{\rho 1}=0
$$

In the equation above, $H_{f}$ is the transmit sensitivity of the coil. Note that B1+ is used commonly in the literature to refer to transmit sensitivity. In this work, we use $H_{f}$, which is defined in the phasor domain and proportional to $\mathrm{B} 1+. E_{z}, E_{\phi}$, and $E_{\rho}$ are the longitudinal, angular, and radial components of the electric field, respectively; $\omega$ is the Larmor frequency; $\mu_{0}$ is the permeability; $\phi$ and $\rho$ are the angular and radial coordinates in the cylindrical coordinate system, respectively; and $j$ is the imaginary number defined by $\sqrt{-1}$.

For this field distribution, the electric field is equal to zero on the entire $\phi=0$ plane. This field can be generated by a linear birdcage coil that has a feed point at the $\phi=\pi / 2$ plane. Similarly, a linear birdcage coil with a feed point at $\phi=0$ and a zero-electric-field plane at $\phi=\pi / 2$ has a field distribution as in the following expression:

$$
E_{z 2}=-H_{f} j \omega \mu_{0} \rho \cos \phi, E_{\phi 2}=0, E_{\rho 2}=0
$$

To obtain a zero-electric-field plane at $\phi=\phi_{0}$, the electric field distribution should be equal to the following expression:

$$
E_{z}=-H_{f} j \omega \mu_{0} \rho \sin \left(\phi-\phi_{0}\right), E_{\phi}=0, E_{\rho}=0
$$

Using simple trigonometric identities, the above distribution can be expressed as the weighted sum of the two distributions in Eqs. 1 and 2, where $E_{z}=\cos \phi_{0} E_{z 1}+\sin \phi_{0} E_{z 2}$.

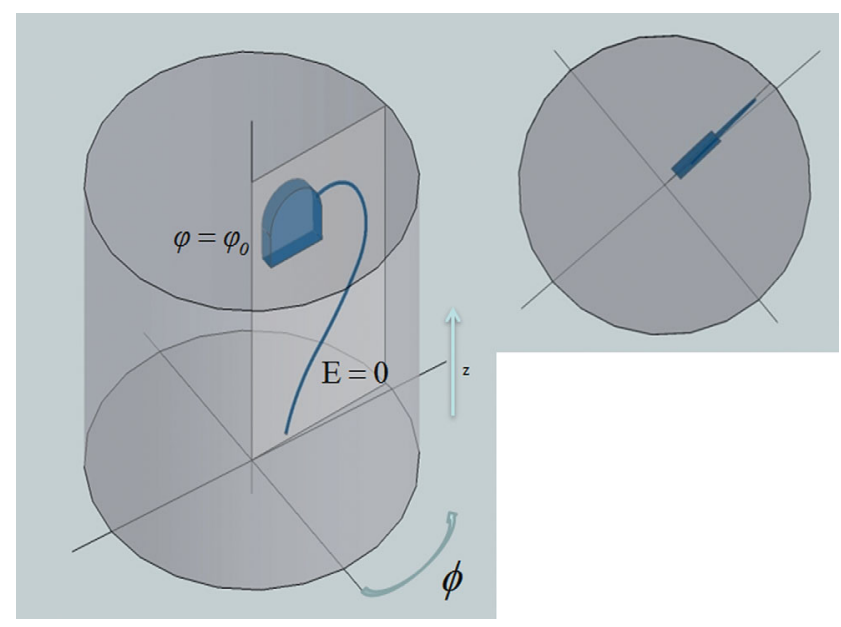

FIG. 1. A metallic lead geometry that is confined inside the $\phi=\phi_{0}$ plane experiences zero electric field. Therefore, no current flows on the lead conductor (Panel a). Similarly, for leads that extend slightly out of the zero-electric-field plane, the induced current on the lead can be made very small (Panel b). A Metallic lead may have a shape that is confined in a large cylindrical volume. In that case, linearly polarized excitation may be insufficient to completely reduce the tip current (Panel c). [Color figure can be viewed in the online issue, which is available at wileyonlinelibrary.com.]

To approximately realize this field, a dual-drive birdcage coil can be used. Let the fields generated by channel 1 and channel 2 be expressed by Eqs. 1 and 2, respectively. Consequently, the field distribution shown in Eq. 3 can be realized by weighting the excitation currents of channel 1 and channel 2 with $\cos \phi_{0}$ and $\sin \phi_{0}$. In this case, a metallic lead with a shape that is confined inside the $\phi=\phi_{0}$ plane will experience zero electric field (Fig. 1). Therefore, no current will flow on the lead conductor. Similarly, for leads that extend slightly out of the zero-electric-field plane, the induced current on the lead can be made small.

This result can also be viewed from a different perspective by exploiting the linearity between the induced current and the excitation voltages. Let the currents induced on the lead tip due to the excitations of channel 1 and channel 2 be $I_{1}$ and $I_{2}$, respectively. Let $\theta$ be a parameter that determines the dual drive channel weightings. Consequently, the total current due to a linear excitation using angle $\theta$ is equal to $I=\cos \theta \cdot I_{1}+\sin \theta \cdot I_{2}$. In this case, by choosing $\theta=\tan ^{-1}\left(-I_{1} / I_{2}\right)$, the current near the lead tip can be set to zero. Note that this solution requires that $I_{1}$ and $I_{2}$ have the same phase.

The phase difference between $I_{1}$ and $I_{2}$ depends on numerous factors, including electromagnetic parameters of the medium and the orientation and the position of the lead with respect to the coil. The electric field due to a perfectly linear excitation has a constant phase in any angular plane as implied in Eq. 3. Therefore, when a lead that is confined in a cylindrical plane is excited with linear excitation, $I_{1}$ and $I_{2}$ have the same phase. Note that the cylindrical plane is defined by the set of points satisfying $\phi=\phi_{0}$ for an arbitrary $\phi_{0}$ in the cylindrical coordinate system. Alternatively, when the leads are not confined in cylindrical planes but in large cylindrical volumes, $I_{1}$ and $I_{2}$ may have different phases. 
Therefore, linearly polarized excitation may be insufficient to completely reduce the tip current. In that case, the value of $\theta$ would be complex, and a different polarization would be required to cancel the tip current. Consequently, to cancel the lead current, it is necessary to adjust the relative phase and magnitudes of the excitation currents.

From an electromagnetic field distribution perspective, if a lead is not confined to the zero-electric-field plane, it could experience electric fields that are high enough to induce currents in the lead tip. In that case, a linear excitation may not be sufficient to completely cancel the lead tip current.

Under all lead placement conditions, the induced current near the tip of a metallic lead can be made to be zero by using a two-channel excitation.

\section{Monitoring Radiofrequency Current-Induced Artifacts}

To monitor artifact intensity, a relation between the magnitude of RF-induced current near metallic implants and the resulting artifact signal was devised.

For a gradient echo (GRE) sequence, the signal intensity depending on the flip angle $\alpha$ can be written as follows (6):

$$
S=\frac{C \cdot \sin (\alpha) \cdot\left(1-e^{-\mathrm{TR} / T_{1}}\right)}{1-e^{-\mathrm{TR} / T_{1}} \cos (\alpha)}
$$

In this equation, $T_{1}$ and TR are the longitudinal relaxation parameter of the tissue and the repetition time of the GRE, respectively. $c$ is a factor that represents the local spin density, the sensitivity of the receiver coil, $T_{2}$ and $T_{2}^{*}$ effects.

To obtain linear excitation, the currents on the two channels of the birdcage coil should be weighted with $\cos \theta$ and $\sin \theta$, where $\theta$ is an arbitrary angle in the interval $[0, \pi]$. Let $I\left[\theta, \phi_{0}\right]$ denote the relationship between $\theta$ and the tip current of the metallic lead located in the $\phi$ $=\phi_{0}$ plane. If the off-set flip angle due to the RF coil excitation is small, then the flip angle near the lead tip will be linearly dependent on the induced current; hence, $\alpha=A \cdot I\left[\theta, \phi_{0}\right] . A$ is a constant that depends on different parameters including the orientation of the lead and the distance of the point where the flip angle is measured.

If the flip angle $\alpha$ due to the RF-induced current is also small by substituting $\sin \alpha \approx \alpha$ and $\cos \alpha \approx 1$ in Eq. 4 , the signal intensity can be approximated with the following expression:

$$
S=c \cdot A \cdot I\left[\theta, \phi_{0}\right]
$$

Equation 5 shows that, to minimize the RF current at the tip of a lead located in the $\phi=\phi_{0}$ half plane, artifact of the current should be minimized by choosing the appropriate dual-drive excitation angle $\theta$. By monitoring the magnitude of the RF current artifact near the lead tip, the excitation pattern that satisfies the above condition can be determined. Signal measurement should be performed close to the lead tip, and at the same time, overflipping should always be avoided to ensure linearity between artifact signal magnitude and the induced current on the lead.

\section{Determining a Safe Excitation Pattern}

The magnitude of the artifact signal intensity due to a linear excitation can be written as follows:

$$
S=\left|S_{1} \cos \theta+S_{2} \sin \theta e^{j \beta}\right|
$$

In this equation, $S_{1}$ and $S_{2}$ are the magnitudes of the induced current artifact signals due to the excitations of channel 1 and channel 2, respectively. $\beta$ is the phase difference between induced currents $I_{1}$ and $I_{2}$. To determine a safe excitation pattern, the total signal intensity $S$ should be minimized. For this purpose, $S_{1}, S_{2}$, and $\beta$ should be estimated by measuring $S$ for different $\theta$ values. The $S_{1}, S_{2}$, and $\beta$ values that minimize the mean square error between the estimated and measured signal intensities were calculated. The mean square error was calculated by calculating the mean square difference between the estimated and measured signal intensities for different $\theta$ values. Once $S_{1}, S_{2}$, and $\beta$ are known, the channel 1 and channel 2 excitation currents can be weighted with $\cos \theta_{0}$ and $\sin \theta_{0} \cdot e^{-j \beta}$ to achieve safe excitation, where $\theta_{0}=\tan ^{-1}\left(-S_{1} / S_{2}\right)$.

\section{EXPERIMENTS}

\section{Phantom Experiments}

To demonstrate the proposed theory, phantom experiments with copper wires and a commercial DBS lead (Medtronic 33877 DBS electrode, Medtronic Inc., Minneapolis, MN) were performed. A phantom of $20 \mathrm{~cm}$ in diameter and $30 \mathrm{~cm}$ in length was prepared with commercially available gel (Dr. Oetker Jello, Izmir, Turkey). To measure the conductivity and relative permittivity, a method based on cylindrical transmission line setup measurements was used. A conductivity of $0.5 \mathrm{~S} / \mathrm{m}$ and a relative permittivity of 70 were obtained with $2.5 \mathrm{~g} / \mathrm{L}$ of salt in the gel solution at $123 \mathrm{MHz}$ (8).

Copper wires and the DBS lead were placed in the phantoms, as shown in Fig. 2. As demonstrated in the figures, the copper wire and the DBS lead had a shape that slightly extended out of the cylindrical angular plane. As shown in Fig. 2c, a copper wire with a helical geometry was used, which was confined in a larger cylindrical volume.

The body coil of the Siemens 3.0 T Trio system was used in all experiments as a dual-drive birdcage coil. First, the body coil (high-pass birdcage) was calibrated by correcting the amplitudes and phases to realize a forward-circular polarized (4) birdcage mode during the experiments. As soon as the amplitudes and phases were calibrated, different polarizations could be obtained. Accordingly, the dual-drive birdcage coil was used in the linear mode. The phantoms with copper wire and a DBS lead were scanned using a GRE sequence with a TR of $200 \mathrm{~ms}$. The excitation voltages were adjusted to obtain a mean flip angle of $2^{\circ}$ in the transverse imaging plane of the phantom. To monitor the magnitude of the artifact due to the RF current near the lead tip, a 

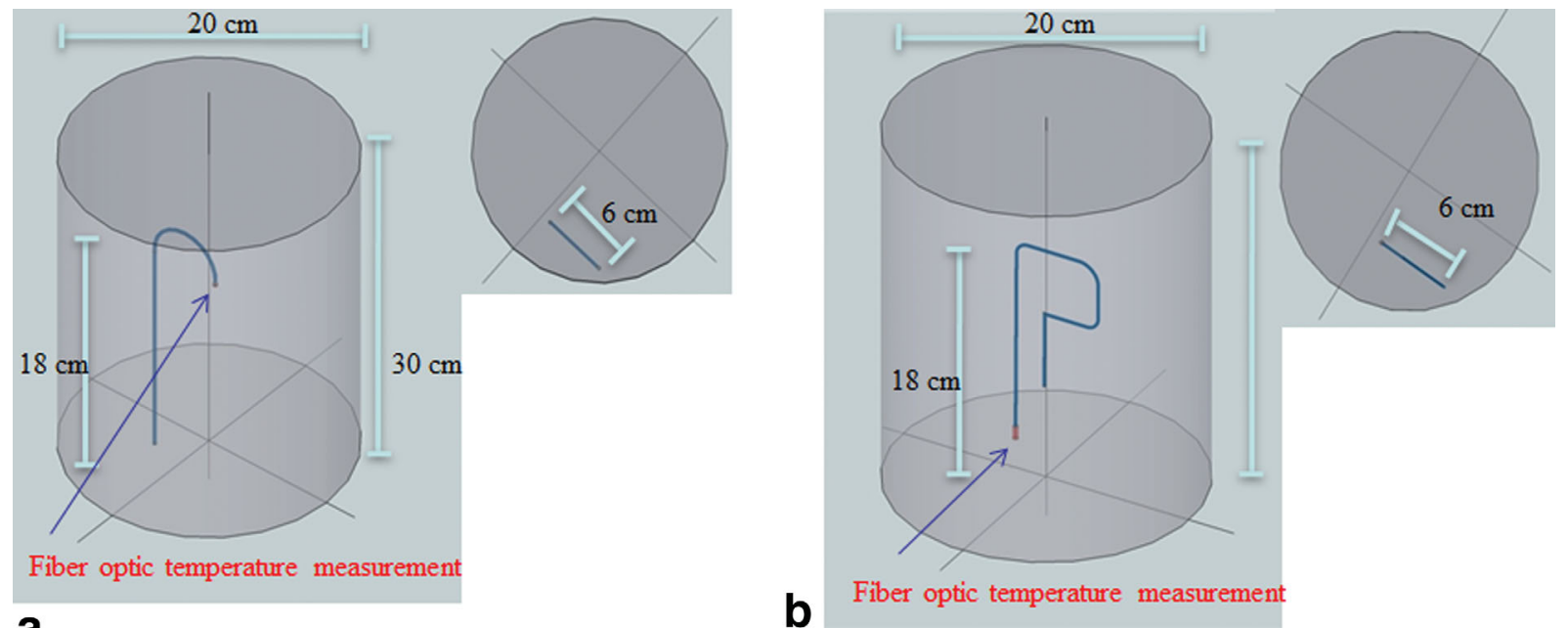

a

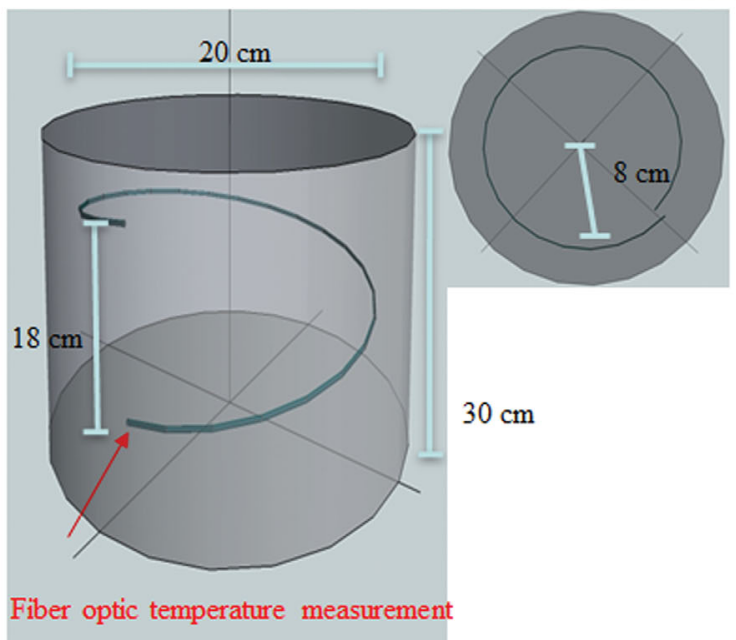

FIG. 2. Lead geometries were not confined in the cylindrical angular planes. Copper wire and a DBS lead in Panel a and Panel b have a geometry that slightly extends out of the cylindrical angular plane. The copper wire in Panel C is confined in a larger cylindrical volume. [Color figure can be viewed in the online issue, which is available at wileyonlinelibrary.com.]

transverse image was obtained. For all cases, a small flip angle and a large TR were chosen so that the SAR value of the sequence was kept at a low level, and no significant heating was expected near the lead tips.

The sequences were run manually using different linear excitation patterns with the dual-drive coil. The amplitudes of the currents in channel 1 and channel 2 were weighted with $\cos \theta$ and $\sin \theta$ to $\operatorname{control}$ the electric field distribution inside the phantom. The value of $\theta$ was varied manually within the interval $[0, \pi]$ with a step size of $\frac{\pi}{36}$. A total of 37 images were obtained for each experiment. In every image, a region of interest of $2 \mathrm{~cm}$ in diameter was selected around the lead tip to measure the maximum magnitude of the RF current artifact (see Fig. 3, Panel a). Measurements were repeated for the copper wire and the DBS leads as explained above.

For all experiments, the safest two-channel excitation pattern was found. This pattern was used to scan the phantom with a high SAR sequence. A GRE sequence with a $4 \mathrm{~ms}$ TR was used for this purpose. For temperature measurements, a signal conditioner (Neoptix ReFlex) with fiber optic temperature sensors (Neoptix
Inc., Quebec City, Canada) was used. The temperature variations at the wire/lead tips were recorded.

After the scan, the gel phantom was kept in the refrigerator for $10 \mathrm{~min}$ to ensure that the temperature reached a steady state of $5.5^{\circ} \mathrm{C}$. From our experience, over certain temperatures $\left(25-30^{\circ} \mathrm{C}\right)$, gel can lose its solid form and turn into a liquid. To eliminate the effects of this condition on temperature measurements, all phantom experiments started at a low initial temperature. Afterward, the phantom was placed in the scanner, and the channel currents were adjusted to obtain standard quadrature excitation. For this purpose, the magnitude of the currents on channel 1 and channel 2 were weighted with unity, and the phases were set to 0 and $\pi / 2$, respectively. Subsequently, the phantom was scanned once more with the quadrature excitation pattern. The temperature variations near the lead/wire tips were recorded similarly. The experiments were repeated with the copper wire and DBS leads with the configurations shown in Fig. 2.

To calculate the maximum local SAR that quadrature excitation causes in a gel phantom (no metallic leads), temperature measurements were performed near the 


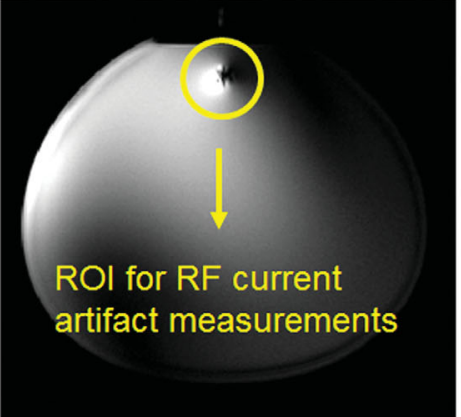

a

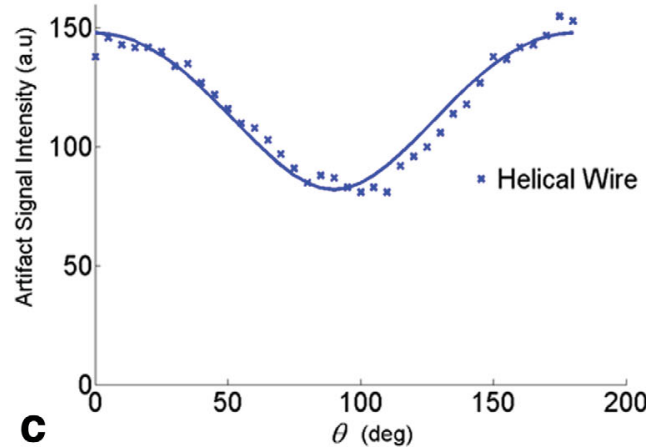

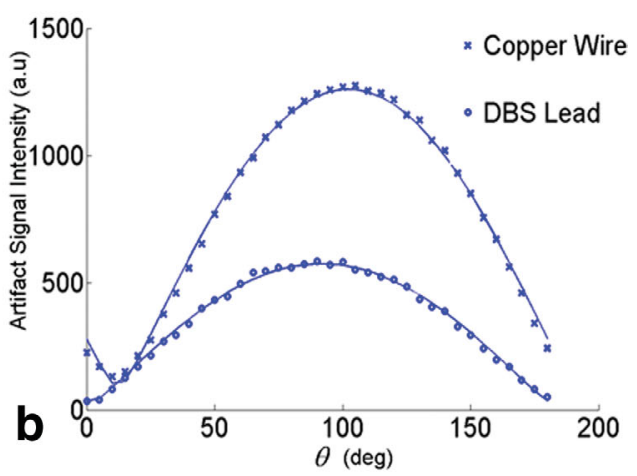

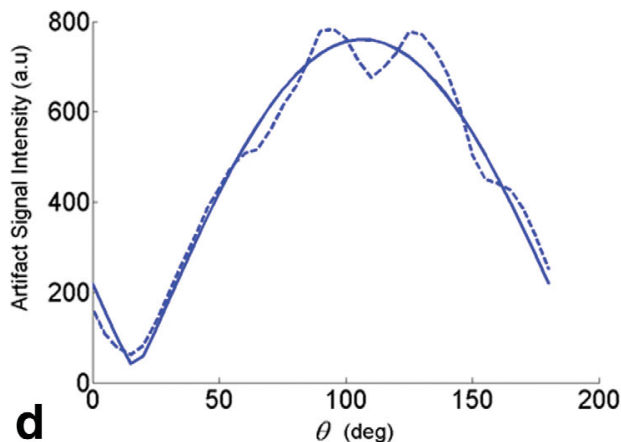

FIG. 3. Panel a shows the induced current artifact near the lead in a phantom. The region of interest that is used to measure the maximum level of the image artifact (bright region) is also presented. The image in Panel a is obtained with a flip angle of $25^{\circ}$ to clearly show the position of the artifact in the phantom Estimated and measured signal intensity curves of copper wire and the DBS lead, which are shown in Fig. 2, Panel a, Panel b, and Panel c, can be seen in Panel b and Panel c. The signal intensity curve for animal experiment can be seen in Panel d. All quantitative artifact signal intensity measurements were made with a $2^{\circ}$ flip angle excitation. [Color figure can be viewed in the online issue, which is available at wileyonlinelibrary.com.]

surface of the phantom. In cylindrical phantoms, peak SAR (maximum SAR in space) occurs close to the surface. For this reason, our SAR measurements were performed near the surface. To calculate the peak SAR caused by a quadrature excitation, the initial slope of the temperature rise at a depth of a $1 \mathrm{~cm}$ from the phantom surface was measured and then multiplied by the specific heat capacity of the gel. The heat capacity of the gel was determined to be $4100 \mathrm{~J} / \mathrm{kg} /{ }^{\circ}$ using the KD2 Pro Thermal Properties Analyzer (Decagon Devices Inc., WA). The peak SAR was calculated as $4.4 \mathrm{~W} / \mathrm{kg}$.

\section{Animal Experiments}

To demonstrate the effectiveness of the method, an ex vivo swine experiment was performed. A copper wire was placed under the skin and muscle tissue immediately after the animal was euthanized by injection of xylazine (2 mg/ $\mathrm{kg}$ ) and ketamine (10 mg/kg). The dimensions of the wire were the same as those of the wire used in the phantom experiment. First, a rectangular region $\sim 1 \mathrm{~cm}$ deep was cut in the abdominal wall of the animal. Subsequently, the copper wire and temperature probe were placed under the cut section, as shown in Fig. 4. Finally, the muscle and skin layer were sewn back in place to cover all parts of the copper wire with living tissue.

The MRI experiments were started immediately after the placement of the wire and temperature probes. The same procedures used for the copper wire-phantom experiments were repeated for the animal experiment.

\section{Volunteer Experiments}

To prove that the method can be used to obtain homogenous flip angle distribution, the safe excitation patterns found in phantom and animal experiments in Sections Animal Experiments and Volunteer Experiments were

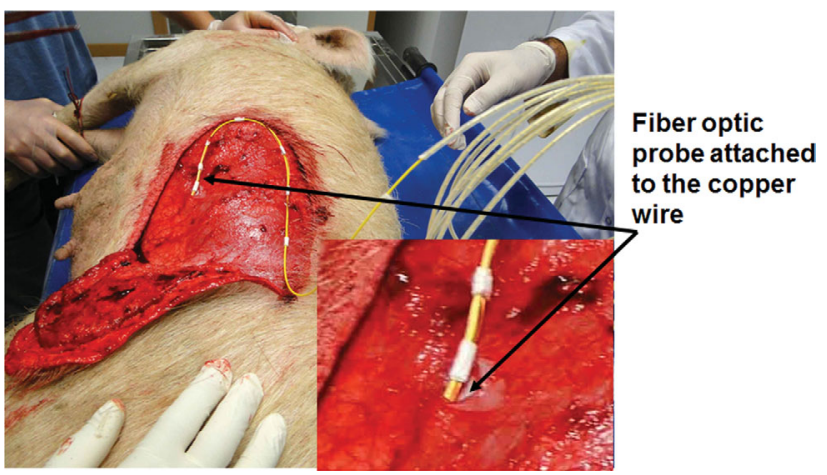

FIG. 4. A rectangular region $\sim 1 \mathrm{~cm}$ deep was cut below the chest of the animal (ex vivo). Subsequently, the copper wire and the temperature probe were placed under the cut section. Finally, the muscle and the skin layer were sewn back in place to cover all parts of the copper wire with animal tissue. [Color figure can be viewed in the online issue, which is available at wileyonlinelibrary.com.] 
Table 1

The Maximum Tip Temperature Is Shown for Each Experiment

\begin{tabular}{lcc}
\hline & $\begin{array}{c}\text { Dual drive } \\
\text { birdcage } \\
\text { excitation }\left({ }^{\circ} \mathrm{C}\right)\end{array}$ & $\begin{array}{c}\text { Quadrature } \\
\text { excitation }\left({ }^{\circ} \mathrm{C}\right)\end{array}$ \\
\hline Copper wire (Phantom) & 0.2 & 13.8 \\
DBS lead & 0.2 & 12.7 \\
Helical copper wire & 0.3 & 4.9 \\
Copper wire & 0.2 & 5.5 \\
$\quad$ (animal experiment) & & \\
\hline
\end{tabular}

Notably, using the safest excitation pattern reduced the tip temperature substantially with respect to that of the quadrature excitation. The error for the temperature data is $\pm 0.1^{\circ} \mathrm{C}$.

used to scan volunteers. For this purpose, the channel 1 and channel 2 excitation currents were weighted with $\cos \theta_{0}$ and $\sin \theta_{0} \cdot e^{-j \beta}$ by using the $\theta_{0}$ and $\beta$ values obtained from the phantom experiments.

For a given imaging plane, the flip angle maps are produced by using the method of actual flip angle imaging (9). The image intensities obtained by using the same flip angle, but different repetition times TR1 and TR2 are used for calculating the flip angle distribution within the single imaging plane. The ratio of TR2 and TR1 were taken as 4 . The resulting maps are compared with the map obtained with a standard quadrature pattern. The normalized root mean square error is calculated for quadrature and safe excitation patterns as a measure of flip angle homogeneity. To calculate the normalized error, the ratio of the standard variation and the mean of the flip angle distribution were calculated. The calculations were made by using single slice, masked magnitude head images.

\section{RESULTS}

\section{Phantom Experiments}

Figure 3 shows the estimated and measured signal intensity curves with respect to $\theta$ for copper wire and DBS leads. The calculated $\beta$ values for the DBS lead, copper wire, and helical DBS lead were found to be $-10,16$, and $85^{\circ}$, respectively. Notably, the $\beta$ values for the copper wire and DBS lead were close to zero, which indicates that, practically, the safest two-channel excitation is the linear excitation for these lead geometries. Furthermore, the minimum point for the artifact intensity curves for these experiments was close to zero. However, for the helical lead experiment, the $\beta$ value was much different than 0 , indicating that the safest two-channel excitation is elliptically polarized for this lead geometry. The $\theta_{0}$ values for copper wire, DBS leads, and helical copper wire experiments were obtained as 5, 20, and $60^{\circ}$, respectively.

The maximum tip temperature for each phantom experiment obtained using safe and quadrature excitation patterns is shown in Table 1 .

\section{Animal Experiments}

Animal experiments were performed on a pig, as described in Section Animal Experiments. Similarly, the maximum magnitude of the RF current artifact inside the region of interest was plotted with respect to $\theta$ in Fig. 3 . The $\beta$ value was calculated to be $-10^{\circ}$ for this experiment. Because $\beta$ is almost zero, linear excitation can be used as the safest excitation pattern in this condition. The minimum current artifact was obtained with the value of $\theta=15^{\circ}$. The pig was scanned using the safest excitation pattern and with the quadrature excitation pattern using the same high SAR sequences that were used in the phantom experiments. Table 1 shows the maximum temperature increase in the lead tip for the quadrature and safe excitation patterns for both the animal experiments. The tip temperature was reduced substantially with the safest excitation patterns compared with quadrature excitation.

\section{Volunteer Experiments}

Flip angle maps obtained with the quadrature and the safe excitation patterns are shown in Fig. 5. Panel a shows the image obtained by a quadrature excitation. Using the safe excitation patterns did not disturb the homogeneity of the flip angle distribution significantly. The normalized root mean square error values were found to be $7.9,7.5,8.2$, and $9.5 \%$ for the quadrature pattern and the safe excitation patterns using $\theta=5, \beta=$ $-10^{\circ}$ (Panel b), $\theta=20, \beta=16^{\circ}$ (Panel c), and $\theta=60, \beta$ $=85^{\circ}$ (Panel d), respectively.

\section{DISCUSSION AND CONCLUSIONS}

A method is herein proposed to reduce the RF heating of the metallic devices using a dual-drive birdcage coil.
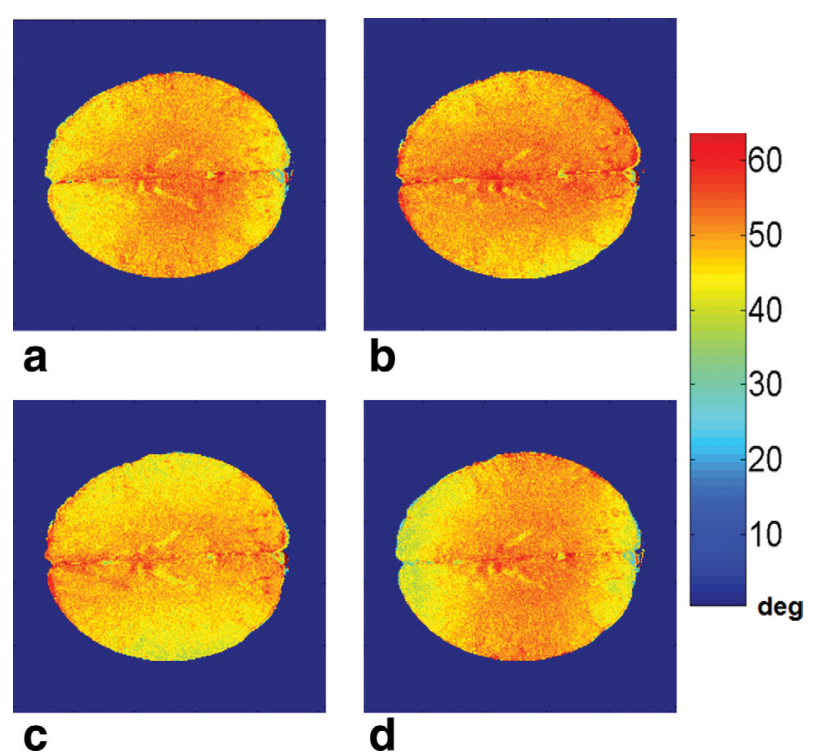

FIG. 5. The flip angle distributions obtained with the quadrature and the safe excitation patterns are shown. The mean of the flip angle distribution were calculated for these excitations as 48.5 , $50.1,47.3$, and $47.9^{\circ}$ respectively. The normalized root mean square error values were calculated as $7.9,7.5,8.2$, and $9.5 \%$, respectively. The calculations were made by using single slice, masked magnitude head images (region of interest covers the entire head image). 
The proposed method was tested with phantom and animal experiments. In all cases, the increase in the tip temperature at the end of the metallic devices was reduced to $0.3^{\circ} \mathrm{C}$.

A GRE sequence was used to measure the RF current artifacts near the lead tips. By changing $\theta$ at intervals of $\pi / 36$ within the range of $[0, \pi]$, a safe excitation pattern was found. Each sequence lasted for $12.8 \mathrm{~s}$, and the total of 37 sequences lasted for $8 \mathrm{~min}$. The entire procedure lasted 12-15 $\mathrm{min}$ in total, because the procedure was not automated and the excitation patterns were entered into the system manually. A constant step size for $\theta$ was used to determine the safe excitation parameters $\beta$ and $\theta_{0}$. To achieve a substantial reduction in measurement time, $\theta$ can be covered in a smaller range or with a variant angular step size.

After the safest two-channel transmit excitation pattern is found, the same pattern can be used in other sequences if the position of the metallic device is not changed. This condition would be valid for patients with implants, given that the location of the implant does not change with respect to the coil during the scan. For interventional procedures, in which the device is bound in a thin cylindrical slice and for which the location is known prior to the scan, a single excitation pattern could be adequate.

A flip angle of $2^{\circ}$ is used for the GRE sequence for measuring the RF current artifacts. Over-flipping the spins near the lead should always be avoided to accurately identify the safe excitation pattern. If required, flip angles lower than $2^{\circ}$ can be used for the low SAR prescans. A close match between estimated and measured signal intensity data should be obtained to accurately calculate the safe excitation pattern.

The method was tested in an ex vivo animal experiment, and the results showed that the tip temperature can be reduced in the presence of tissue inhomogeneities. However, the effect of motion artifacts should be considered for clinical studies. Respiratory-gated sequences can be used for this purpose.

In some cases, the magnetic field due to the current may be in the longitudinal direction. To measure the artifact signal, an imaging plane should be chosen such that the induced magnetic field has a nonzero transverse component in that plane. In this study, the imaging plane was always chosen as the transverse plane. For different lead orientations, the transverse plane may not always be the most appropriate imaging plane.

By determining the safest dual-drive excitation pattern, the current near the tip of the lead is minimized. At another location on the lead, the RF current may be nonzero. The tip point is the most critical point where the temperature should be minimized. The other end of the lead is either terminated with the implant case or simply extends outside the body, as in interventional procedures. The local SAR at the shaft of the device/lead is also significantly less than the tip SAR, as reported in a previous study (10). Therefore, in this study, the effect of minimizing the current and temperature near the lead tip was investigated.

The comparison of the tip temperature due to safe dual-drive excitation and the quadrature excitation may depend on the position of the implant. Implants near the surface experience the largest electric field. For this reason, temperature measurements were made with leads that were located near the surface. For different lead geometries, it was demonstrated that a modified dual-drive excitation pattern can reduce the RF heating at the tip of the lead. In a previous study (4), heating problems of lead geometries that are perfectly confined in cylindrical planes (Fig. 1) were investigated. In this work, it is demonstrated that lead geometries that are not necessarily confined in cylindrical planes can be handled with a modified dual-drive excitation pattern (Fig. 2).

The tip heating can also be reduced when the transverse electric field components are present in the body as a result of finite body/coil dimensions and tissue inhomogeneities. However, the type of the excitation pattern that reduces the heating may depend on various factors, such as the lead geometry and the relative position of the patient with respect to the RF coil (landmark position).

The use of linear excitation enabled the preservation of the flip angle distribution with respect to quadrature excitation in the center of the head. However, twochannel excitations aside from quadrature or linear excitation may cause reductions in flip angle and RF field homogeneity. This effect can be more significant in higher field strengths. Furthermore, an excitation aside from a quadrature excitation may cause greater average and local SAR in the body. The proposed method can be extended to parallel transmission to take advantage of the increased freedom provided by a greater number of transmission elements (11). Different parallel transmission techniques may be used to allow local and average SAR reduction while preserving flip angle homogeneity (12).

We demonstrated a method to reduce the RF heating of metallic devices with a dual-drive birdcage coil. The artifacts that result from RF-induced currents on the metallic leads were monitored to determine the safest excitation pattern with a dual-drive birdcage coil. As a result, heating at the tip of the metallic devices was reduced significantly, as shown by both phantom and animal experiments.

\section{REFERENCES}

1. Nitz WR, Oppelt A, Renz W, Manke C, Lenhart M, Link J. On the heating of linear conductive structures as guide wires and catheters in interventional MRI. J Magn Reson Imaging 2001;13: 105-114.

2. Yeung CJ, Susil RC, Atalar E. RF safety of wires in interventional MRI: using a safety index. Magn Reson Med 2002;47:187-193.

3. Gray RW, Bibens WT, Shellock FG. Simple design changes to wires to substantially reduce MRI-induced heating at $1.5 \mathrm{~T}$ : implications for implanted leads. Magn Reson Imaging 2005;23:887-891.

4. Eryaman Y, Akin B, Atalar E. Reduction of implant RF heating through modification of electric field. Magn Reson Med 2011;65: 1305-1313.

5. Nordbeck P, Weiss I, Ehses P, Ritter O, Warmuth M, Fidler F, Herold V, Jakob PM, Ladd ME, Quick HH, Bauer WR. Measuring RF-induced currents inside implants: impact of device configuration on MRI safety of cardiac pacemaker leads. Magn Reson Med 2009;61: 570-578.

6. van den Bosch MR, Moerland MA, Lagendijk JJW, Bartels LW, van den Berg CAT. New method to monitor RF safety in MRI-guided interventions based on RF induced image artefacts. Med Phys 2010; 37:814-821. 
7. Etezadi-Amoli M, Stang P, Zanchi MG, Pauly JM, Scott GC, Kerr AB. Controlling Induced Currents in Guidewires Using Parallel Transmit. In Proceedings of the 18th Annual Meeting of ISMRM, Stockholm, Sweden, 2010. p. 777.

8. Akin B, Eryaman Y, Atalar E. A method for phantom conductivity and permittivity measurements. In Proceedings of the 26th Annual Meeting of ESMRMB, Antalya, Turkey, 2009. p. 518.

9. Nehrke K. On the steady state properties of actual flip angle imaging (AFI). Magn Reson Med 2009;61:84-92
10. Ladd ME, Quick HH. Reduction of resonant RF heating in intravascular catheters using coaxial chokes. Magn Reson Med 2000;43: $615-619$.

11. Eryaman Y, Demir T, Atalar E. Reduction of RF heating of metallic devices using transmit arrays. In Proceedings of the 18th Annual Meeting of ISMRM, Stockholm, Sweden, 2010. p. 3883.

12. Lee J, Gebhardt M, Wald LL, Adalsteinsson E. Local SAR in parallel transmission pulse design. Magn Reson Med 2012;67: 1566-1578. 